\title{
Paget Disease of the Temporal Bone
}

\author{
Fayez Bahmad Jr. and Saumil N. Merchant \\ Otopathology Laboratory, Massachusetts Eye and Ear Infirmary, and Harvard Medical School, \\ Boston, Massachusetts, U.S.A
}

\begin{abstract}
Paget disease of bone is a disorder of remodeling characterized by uncoupling of bone resorption and bone formation (1). It is thought to be caused by a slow virus infection. Paget disease can affect one or multiple bones in the systemic skeleton, including the temporal bone $(1,2)$. Typically, there are three phases of activity in Paget disease affecting the systemic skeleton $(1,2)$. The first or osteolytic phase demonstrates the prominent resorption of bone by numerous large osteoclasts. The second or mixed phase involves both osteoclastic and osteoblastic activities, creating a mosaic appearance typical of Paget disease. The third or osteoblastic phase is characterized by abundant osteoblastic activity and a prominent mosaic pattern. In the temporal bone, a fourth or remodeled phase, where areas of inactive osteoblastic pagetic bone are remodeled into healthier lamellar bone with distinct haversian canal systems, has been described (2).
\end{abstract}

\section{CASE REPORT}

This patient was treated for epistaxis at the age of 82 years and was noted to have a mass involving the maxillary and the ethmoid sinuses. Surgical biopsy at a Caldwell-Luc operation showed Paget disease of bone. Paget disease was also found in the spine, pelvis, clavicles, scapulae, and other bones. The patient complained of a progressive hearing loss beginning at age 78 . An audiogram at age 83 showed a bilateral mixed hearing loss, with bone-conduction thresholds at $30 \mathrm{~dB}$ in the low frequencies and air-conduction thresholds at about 80 to $90 \mathrm{~dB}$. The speech discrimination score was $60 \%$ on the right and $40 \%$ on the left. He died at age 85 as a result of renal and respiratory failure.

The histopathologic findings are similar in both temporal bones. There is extensive pagetic involvement of the otic capsule, mastoid, and petrous apex (Fig. 1). Most of the endochondral bone of the otic capsule is replaced by pagetic bone. However, there is no invasion of the inner ear. The internal auditory canal is elongated because of the pagetic thickening of the petrous bones, but it is widely patent. Microfractures are evident within the otic capsule, especially over the promontory (Fig. 2). There are scattered losses of hair cells in the cochlea, but the organ of Corti is generally present throughout. There is a moderate loss of cochlear neurons compared with that in healthy newborns. The vestibular sense organs seem intact. There is no evidence of pagetic involvement of the ossicles and no evidence of any lesions affecting the middle ear.

\section{DISCUSSION}

Paget disease of the temporal bone is often associated with a mixed hearing loss. There is typically an air-bone gap in the low frequencies with a downsloping, high-frequency sensorineural hearing loss. The pathogenesis for the conductive and sensorineural hearing loss

Address correspondence and reprint requests to Saumil N. Merchant, M.D., Massachusetts Eye and Ear Infirmary, 243 Charles Street, Boston, MA 02114; E-mail: saumil_merchant@meei.harvard.edu. 
is not known. As illustrated in the present case, histopathologic studies have failed to find consistent correlates for the hearing loss (3). Clinical studies using audiometric and computed tomographic analysis have demonstrated a loss of otic capsule bone mineral density correlated with both the high-frequency sensorineural hearing loss and the air-bone gap (4). The air-bone gap has been hypothesized to be the result of the alteration of the mechanics of the ear $(3,4)$. The down-sloping, sensorineural hearing loss has been hypothesized to represent abnormalities of the metabolic homeostasis of the cochlea as a result of the liberation of cytokines from the pagetic bone and the dysfunction of the spiral ligament (5).

\section{References}

1. Pilch, BZ. Head and Neck Surgical Pathology. Philadelphia, PA: Lippincott Williams \& Wilkins; 2001.

2. Schuknecht, HF. Pathology of the Ear. 2. Philadelphia, PA: Lea and Febiger; 1993.

3. Khetarpal U, Schuknecht HF. In search of pathologic correlates for hearing loss and vertigo in Paget's disease. A clinical and histopathologic study of 26 temporal bones. Ann Otol Rhinol Laryngol Suppl 1990;145:1-16. [PubMed: 2106820]

4. Monsell EM. The mechanism of hearing loss in Paget's disease of bone. Laryngoscope 2004;114:598606. [PubMed: 15064610]

5. Adams JC. Clinical implications of inflammatory cytokines in the cochlea: a technical note. Otol Neurotol 2002;23:316-22. [PubMed: 11981388] 


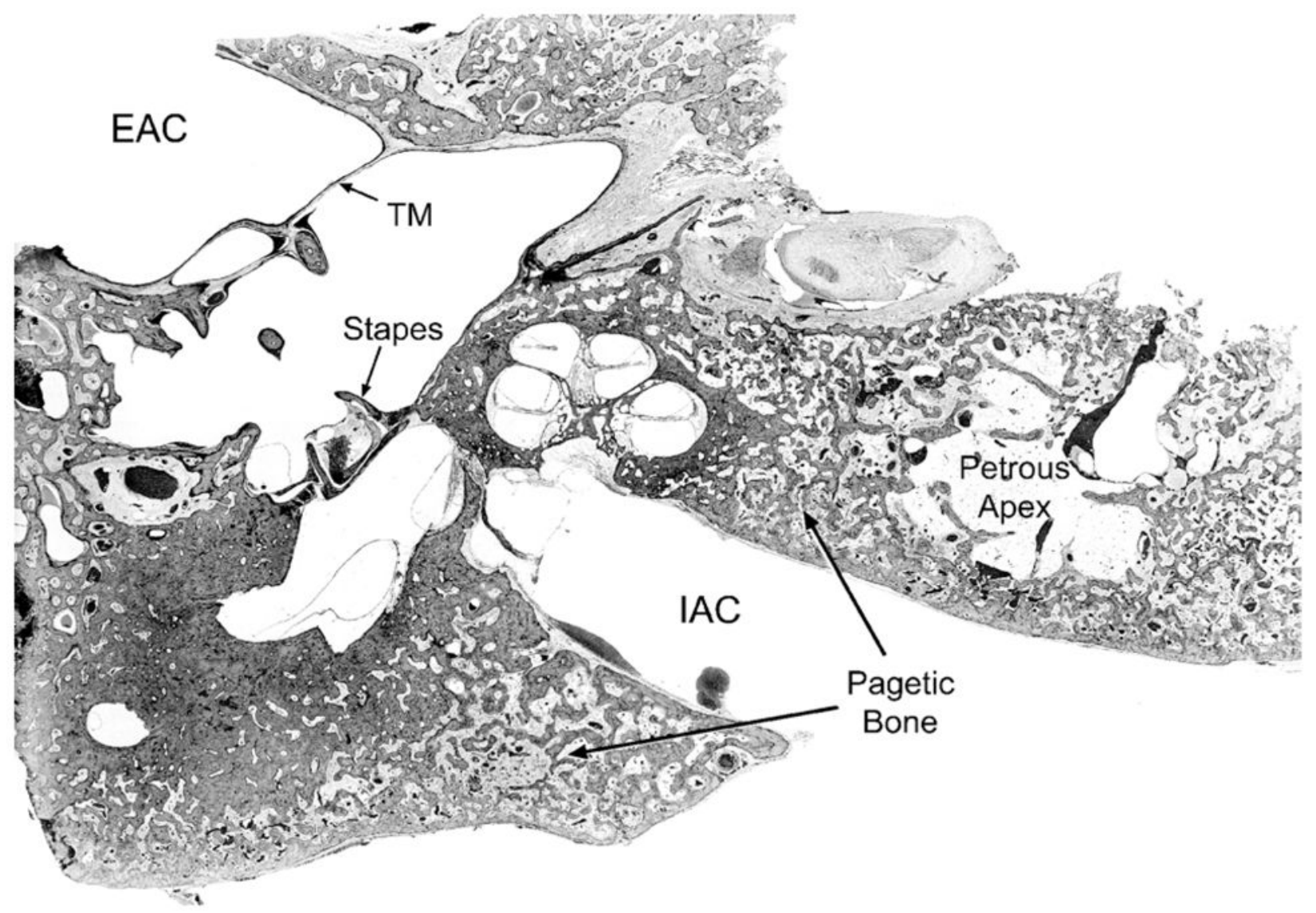

FIG. 1.

Image showing the left temporal bone (original magnification $\times 3.5$ ). Note the extensive pagetic involvement of the petrous apex, otic capsule, and mastoid. The pagetic bone is mainly in the osteoblastic phase and shows the typical mosaic pattern. The petrous apex is greatly thickened by pagetic bone. There is no involvement of the ossicles. EAC indicates external auditory canal; IAC, internal auditory canal; TM, tympanic membrane. 


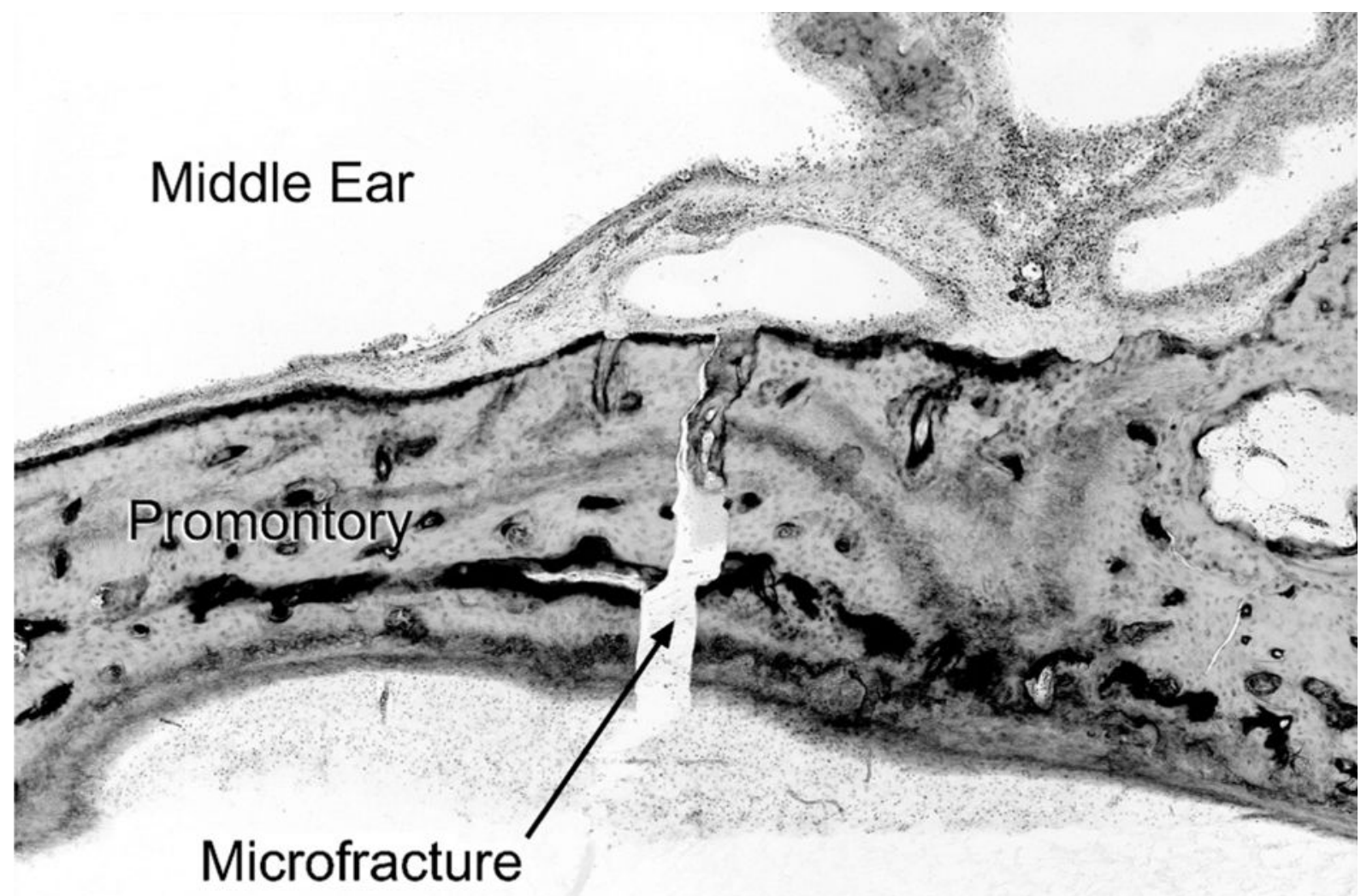

FIG. 2.

High-power field view of right temporal bone showing a microfracture in the promontory (original magnification $\times 55$ ). 